ESAIM: PROCEEDINGS, November 2002, Vol.12, 61-66

M.Thiriet, Editor

\title{
A NONLINEAR ELASTIC SOFT TISSUE MODEL FOR CRANIOFACIAL SURGERY SIMULATIONS
}

\author{
E. Gladilin, S. Zachow, P. Deuflhard and H.-C. Hege ${ }^{1}$
}

\begin{abstract}
Biomechanical modeling of soft tissue is essential to many computer assisted medical applications. Because of complexity of soft tissue behavior and a lack of exact and comprehensive data on material properties of living tissues, simplified models such as widespread linear elastic models are usually applied. However, large deformations often occurring in craniofacial surgery can not be accurately modeled via linear elastic approach, since it is generally limited by the assumption of small deformations. The investigations carried out in the present work shows that the nonlinear elastic model yields more realistic results in simulations of large soft tissue deformation.
\end{abstract}

\section{INTRODUCTION}

The finite element method (FEM) becomes in the last three decades a state of the art approach for the realistic simulation of the soft tissue deformation in the computer assisted surgical applications. However, the consistent physically based modeling of soft tissues is substantially limited by the complexity of their biomechanical properties. In general, soft tissue exhibits anisotropic, nonhomogeneous, nonlinear plastic-viscoelastic mechanical behaviour. The complex models, which consider all these properties, are difficult to implement and to control. Thus, the simplified constitutive models usually have to be applied. The goal of the soft tissue modeling within the scope of craniofacial surgery planning is the 'long term' prediction of the patient's postoperative appearance, i.e., the quasi-static deformation of relatively thin facial tissue under the impact of forces indirectly given by the prescribed displacements of bone structures has to be calculated. Thereby, the deformations are usually performed by surgeons (i) 'at ones', in the case they are small, or (ii) over a longer period of time, if they are large. From this problem definition, a linear elastic model of soft tissue appears a natural approximation. In our previous works [6], we have investigated the scope of validity of a linear elastic model and have found that it produces a substantial error in the case of large deformations. In this work, we present the results of the application of a nonlinear elastic FE-based model, where we primarily focus on the investigation of the effects of the so-called geometrical nonlinearity. Being independent of material properties, this kind of nonlinearity is relevant for any large deformed physical body, including soft tissue.

\section{Nonlinear Elasticity}

In this section, we briefly explain the basics of elasticity theory.

1 Zuse Institute Berlin (ZIB) Takustr.7, 14195 Berlin, Germany, email: \{gladilin,zachow,deuflhard,hege\}@zib.de

(c) EDP Sciences, SMAI 2003 


\subsection{Geometrical Nonlinearity}

Under external forces, physical bodies are deformed, which means that they change both their shape and volume. The deformation of a body is completely described if the displacements $u_{i}$ of all its points are known:

$$
u_{i}=x_{i}^{\prime}-x_{i}
$$

where $x_{i}$ and $x_{i}^{\prime}$ denotes coordinates of a point before and after the deformation. The strain tensor $\varepsilon_{i j}$ that describes the deformation of an infinitesimal volume is generally a nonlinear function of displacements

$$
\varepsilon_{i j}=0.5\left(\partial_{j} u_{i}+\partial_{i} u_{j}+\partial_{i} u_{l} \partial_{j} u_{l}\right)
$$

which is known as geometrical nonlinearity. In the case of small deformations, the derivatives of the displacement vector, which represent the relative displacements, are significantly smaller than 1:

$$
\left|\partial_{j} u_{i}\right| \ll 1
$$

In this case the quadratic term in (2) can be neglected, and the strain tensor can be linearized:

$$
\varepsilon_{i j} \approx e_{i j}=0.5\left(\partial_{j} u_{i}+\partial_{i} u_{j}\right)
$$

Thus, the linearization condition can be formulated as

$$
\epsilon=\max \left(\left|e_{i j}\right|\right) \ll 1
$$

(5) can be used for the monitoring of the local linearization error [6] in a more exact form $\epsilon<\epsilon_{\max }$ with a typical threshold for the maximum linearization error of approximately five percent, i.e. $\epsilon_{\max }=0.05$.

\subsection{Constitutive Equations}

The stress-strain relationship (constitutive equation) depends on the material's properties $\sigma_{i j}=f\left(\varepsilon_{i j}\right)$. In this work, we assume soft tissue an isotropic, homogeneous (Hookean) material with the piecewise linear stressstrain relationship:

$$
\sigma_{i j}=\frac{E}{1+\nu}\left(\varepsilon_{i j}+\frac{\nu}{1-2 \nu} \varepsilon_{l l} \delta_{i j}\right)
$$

where $E$ is the Young's modulus, which describes the stiffness of a material, and $\nu$ is the Poisson's ratio, which describes the material's compressibility. Typical values for Young's modulus are E $\in$ [2, 200]kPa [4]. According to $[5,7]$ the Poisson's ratio lies for nearly incompressible soft tissue close to 0.5 , but depending on particular tissue type, age, sex and other factors it can vary. In this work, we assume $\nu=0.45$.

\subsection{Static Equilibrium State}

In static equilibrium, the sum of external and internal forces vanish. In the case of a pure displacement problem for a St. Venant-Kirhhoff material that is characterized by (6), the boundary value problem (BVP) in the Lagrangian formulation takes the form [2]:

$$
\left\{\begin{array}{l}
\partial_{j}\left(\sigma_{i j}+\sigma_{k j} \partial_{k} u_{i}\right)=0 \text { in } \Omega, \\
u_{i}=c_{i} \text { on } \Gamma_{\text {essential }} \subset \Omega, \\
\sigma_{i j} n_{j}=0 \text { on } \Gamma_{\text {natural }} \subset \Omega,
\end{array}\right.
$$

where $c_{i}$ denotes the predefined displacement on the essential boundary $\Gamma_{\text {essential }} \subset \Omega$ of the domain of interest $\Omega$. 


\subsection{Iterative Solution Scheme}

Because of (2), the mapping $u_{i} \rightarrow \sigma_{i j}$ is nonlinear. Thus, (7) leads to the nonlinear system of equations respectively displacements $\mathbf{u}$ that can be written in the symbolic form

$$
\mathbf{A}(\mathbf{u})=\mathbf{b}
$$

where $\mathbf{A}(\mathbf{u})$ denotes the operator of nonlinear elasticity and $\mathbf{b}$ is the vector of all known loads. For numerical solution of (8), any appropriate iterative scheme can be used. The Newton's method used in this work consists in recursively solving the set of linearized approximations to (8):

$$
\mathbf{A}^{\prime}\left(\mathbf{u}^{\mathbf{n}}\right) \delta \mathbf{u}^{\mathbf{n}}=\mathbf{b}-\mathbf{A}\left(\mathbf{u}^{\mathbf{n}}\right),
$$

where $\delta \mathbf{u}^{\mathbf{n}}=\mathbf{u}^{\mathbf{n}+\mathbf{1}}-\mathbf{u}^{\mathbf{n}}$ is the $n$-th increment of the displacement and $\mathbf{A}^{\prime}\left(\mathbf{u}^{\mathbf{n}}\right)$ denotes the Fréchet derivative of $\mathbf{A}(\mathbf{u})$, the so-called tangent stiffness.

\section{Adaptive Nonlinear Finite Element Approach}

From our findings, the adaptivity of the numerical scheme on different levels of problem solving is essential for the achievement of the efficient and robust performance. The main adaptive features of our approach include the adaptive mesh refinement, the adaptive linear/nonlinear assembly of stiffness matrix as well as the adaptive solving scheme.

To obtain the numerical solution of (7) a finite element method (FEM) is used. The so-called weak formulation of (9), with weighting functions $\psi_{i}$ vanishing on the boundary $\Gamma$ of the domain $\Omega$, takes the form [2]:

$$
\int_{\Omega} C_{i j p q}^{\prime}\left(\nabla \mathbf{u}^{n}\right) \partial_{p} \delta u_{q}^{n} \partial_{j} \psi_{i} d \Omega=\int_{\Omega} \delta f_{i}^{n} \psi_{i} d \Omega,
$$

with

$$
C_{i j p q}^{\prime}\left(\nabla \mathbf{u}^{n}\right)=C_{i j p q}+C_{k j p q} \partial_{k} u_{i}^{n}+C_{i j r p} \partial_{r} u_{q}^{n}+C_{k j p r} \partial_{r} u_{q}^{n} \partial_{k} u_{i}^{n}+C_{p j s r} \varepsilon_{s r}\left(\mathbf{u}^{n}\right) \delta_{i q} .
$$

The solution of (10) yields the $n$-th increment of the displacement vector $\delta u_{i}^{n}$. Thus, the application of the iterative solution scheme results in the successive approximation of the $(n+1)$ st displacement

$$
\mathbf{u}^{n+1}=\mathbf{u}^{n}+\delta \mathbf{u}^{n} .
$$

For each iteration the sparse linear system of equations (10) respectively the $n$-th increment of the displacement is solved with the help of a Jacobi-preconditioned conjugated gradient method.

The conventional nonlinear FEM is computationally heavy. Because of (11), at each iteration step the tangent stiffness matrix has to be assembled anew. Such a full update is very expensive and may be substituted with a weaker formulation, i.e. the simplified Newton's method that is

$$
\mathbf{A}^{\prime}\left(\mathbf{u}^{\mathbf{0}}\right) \delta \mathbf{u}^{\mathbf{n}}=\mathbf{b}-\mathbf{A}\left(\mathbf{u}^{\mathbf{n}}\right) .
$$

In this approach, the most expensive part of the nonlinear FE calculation, the assembly of the tangent stiffness matrix, has to be performed only once.

An additional improvement of performance can be reached by applying the true nonlinear assembly of local stiffness matrix on large deformed elements only. Indeed, if higher order terms in (11) are small in comparison with linear one they can be neglected as it is assumed in linear elasticity. To switch between nonlinear and linear assembly of an elementary stiffness matrix, a criterion based on the monitoring of the local linearization error (3) is used.

Finally, the adaptive mesh refinement based on robust error estimators $[1,3]$ is used, in order to obtain the accurate discrete solution with the user defined precision. 


\section{Geometric Modeling}

Geometrical models of the patient's head are generated from the CT data with help of the multipurpose visualization and modeling system AMIRA, which has been developed at our institute [8]. A final model consists of triangulated surfaces filled up with an unstructured tetrahedral subgrids. The planning of surgical bone rearrangement includes the simulation of splitting or cutting bone segments (osteotomy) and repositioning of separated bony structures. The boundary conditions for the following numerical simulation, are given by the prescribed displacements induced by the rearrangement of bone structures.

\section{Experimental Results}

The nonlinear elastic FE approach is applied for the soft tissue prediction in a clinical case. The Figure 4 illustrates the simulation of a mandible distraction for a juvenile patient with so-called congenital mandibular hypoplasia. The corrective surgical impact consists in the stepwise distraction of mandible. The total maximum displacement of bone structures for this patient amounts up to $40 \mathrm{~mm}$ ! Such extremely large deformation is normally performed by surgeons stepwise over several months with the help of an distractor device. The deformation of facial tissue induced by the repositioning of mandible has been simulated both via the linear and nonlinear elastic FEM. Figure 4 shows the comparison between the facial outline predicted by the linear and nonlinear elastic approach. In comparison with the outcome of the linear elastic simulation, the nonlinear elastic FEM produces effectively smaller displacements, in particular, in the largely deformed chin area. Since postoperative data are not available for this patient, a direct comparison with the simulation result is not possible yet.

In the example above, the deformation for a grid of approximately $5 \times 10^{5}$ tetrahedra has to be computed. The calculation of the linear solution for this grid took 3.1 minutes. The calculation of the nonlinear solution took 9 minutes, which corresponds to 3 iterations of Newton's method. The calculations were performed on PC Pentium PIII with 600MHz. The FE code used for both linear and nonlinear elastic simulations was developed and optimized at our institute.
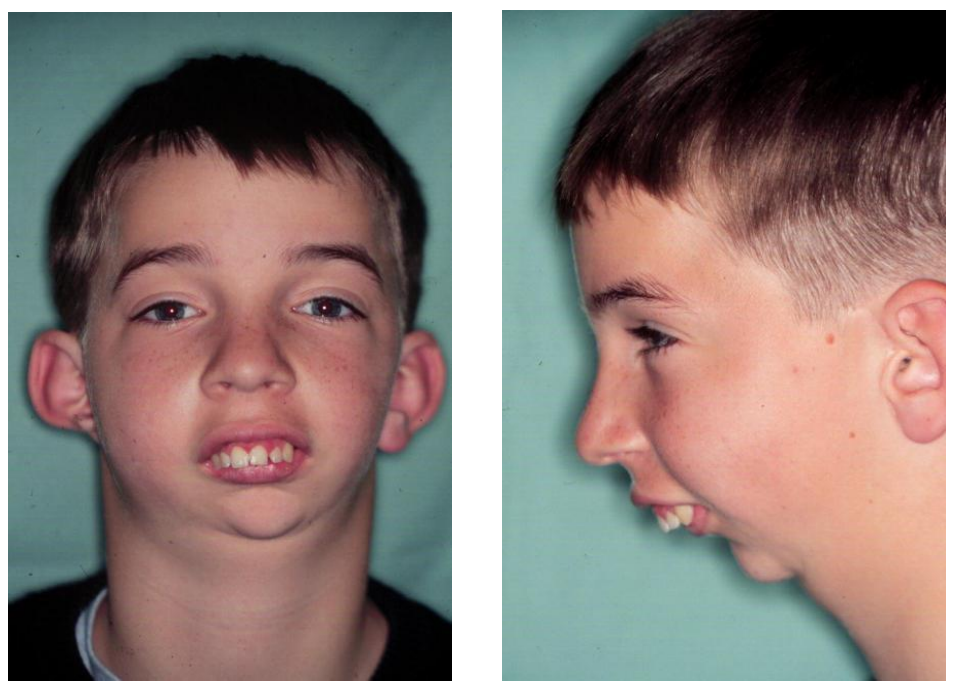

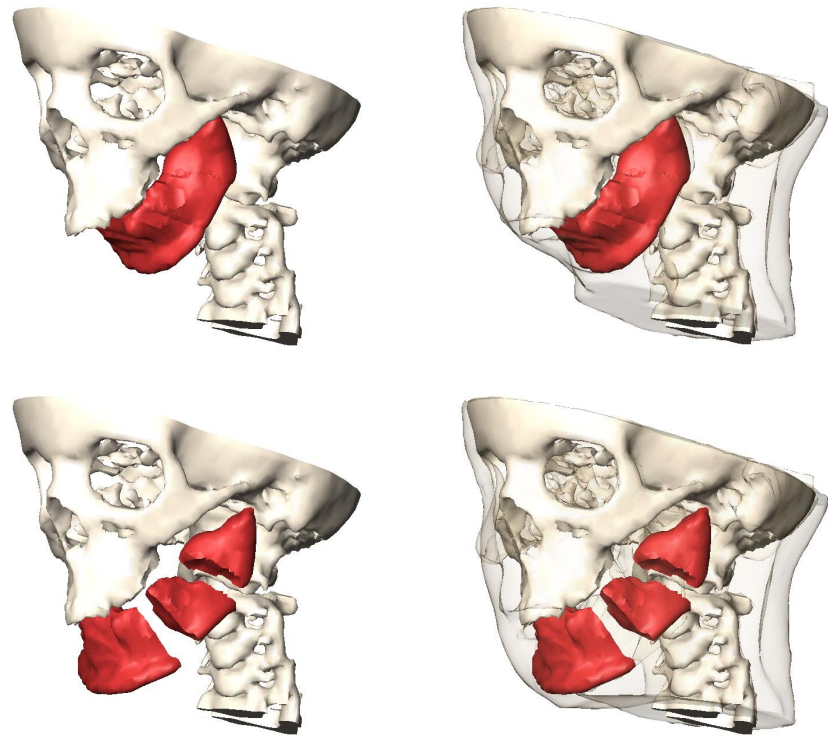

FiguRE1.Simulation of mandible distraction for a patient with congenital mandibular hypoplasia.
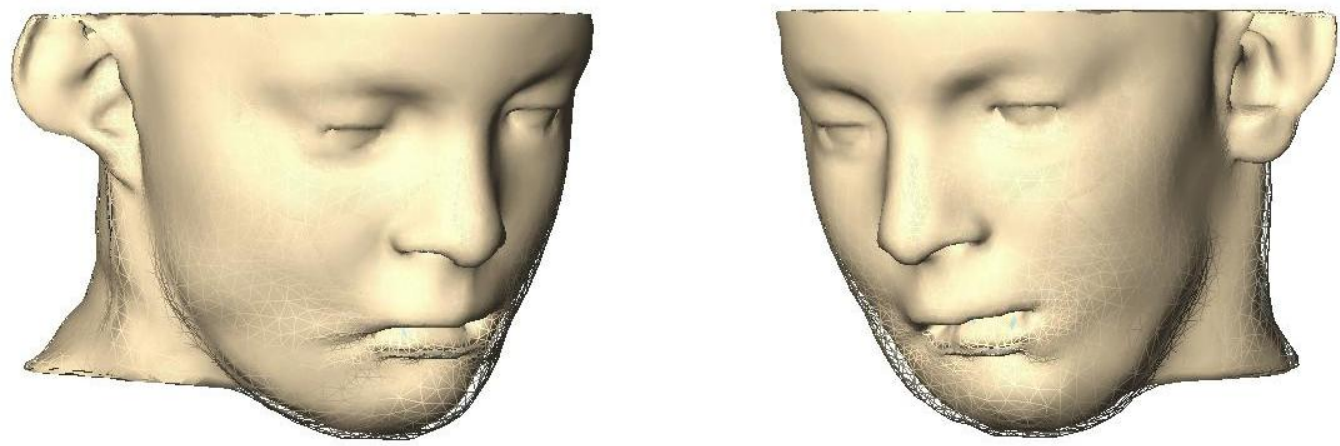

FIGURE 2.Comparison between the facial outline predicted by the linear (outer line mesh) and nonlinear (inner shaded mesh) elastic model.

\section{Conclusion}

In this paper, we present an adaptive nonlinear elastic FE-based approach for soft tissue prediction in craniofacial surgery planning. The comparison between the linear and nonlinear elastic soft tissue model is carried out. It is shown that the nonlinear approach yields more accurate and realistic results especially in large deformed areas and on curved boundaries. The efficient adaptive numerical scheme allows to perform calculations of nonlinear elastic deformations in acceptable timeframe. From our results, the fast linear elastic FEM is suited as a preprocessing for more realistic calculations via the nonlinear elastic FEM.

\section{REFERENCES}

[1] F. A. Bornemann, B. Erdmann, and R. Kornhuber. Adaptive multilevel-methods in three space dimensions. Int. J. for Numer. Meth. in Eng., 36:3187-3203, 1993.

[2] P. G. Ciarlet. Mathematical Elasticity. Volume I: Three-Dimensional Elasticity, volume 20 of Studies in Mathematics and its Applications. North-Holland, Amsterdam, 1988. 
[3] P. Deuflhard, P. Leinen, and H. Yserentant. Concepts of an adaptive hierarchical finite element code. Impact of Computing in Science and Engineering, 1:3-35, 1989.

[4] F. Duck. Physical properties of tissues: a comprehensive reference book. Academic Press, London, 1991.

[5] Y. C. Fung. Biomechanics - Mechanical Properties of Living Tissues. Springer, Berlin, 1993.

[6] E. Gladilin, S. Zachow, P. Deuflhard, and H.-C. Hege. Validation of a linear elastic model for soft tissue prediction in craniofacial surgery. In Proc. of SPIE Medical Imaging Conference, volume 4319, pages 27-35, San Diego, 2001.

[7] N. Özkaya and Nordin M. Fundamentals of Biomechanics. Springer, Berlin, 1999.

[8] D. Stalling, M. Zöckler, and H.-C. Hege. Amira - an advanced 3D visualization and modeling system. URL: http://amira.zib.de. 